\title{
STABLE RATIONAL COHOMOLOGY OF AUTOMORPHISM GROUPS OF FREE GROUPS AND THE INTEGRAL COHOMOLOGY OF MODULI SPACES OF GRAPHS
}

\author{
Craig A. Jensen
}

\begin{abstract}
It is not known whether or not the stable rational cohomology groups $\tilde{H}^{*}\left(\operatorname{Aut}\left(F_{\infty}\right) ; \mathbb{Q}\right)$ always vanish (see Hatcher in $[\mathbf{5}]$ and Hatcher and Vogtmann in [7] where they pose the question and show that it does vanish in the first 6 dimensions). We show that either the rational cohomology does not vanish in certain dimensions, or the integral cohomology of a moduli space of pointed graphs does not stabilize in certain other dimensions. Similar results are stated for groups of outer automorphisms. This yields that $H^{5}\left(\hat{Q}_{m} ; \mathbb{Z}\right), H^{6}\left(\hat{Q}_{m} ; \mathbb{Z}\right)$, and $H^{5}\left(Q_{m} ; \mathbb{Z}\right)$ never stabilize as $m \rightarrow \infty$, where the moduli spaces $\hat{Q}_{m}$ and $Q_{m}$ are the quotients of the spines $\hat{X}_{m}$ and $X_{m}$ of "outer space" and "auter space", respectively, introduced in [3] by Culler and Vogtmann and [6] by Hatcher and Vogtmann.
\end{abstract}

\section{Introduction}

Let $F_{n}$ denote the free group on $n$ letters and let $\operatorname{Aut}\left(F_{n}\right)$ and $\operatorname{Out}\left(F_{n}\right)$ denote the automorphism group and outer automorphism group, respectively, of $F_{n}$. In [5] Hatcher shows that the integral cohomology of the infinite symmetric group $\Sigma_{\infty}$ is a direct summand of the integral cohomology of $\operatorname{Aut}\left(F_{\infty}\right)$. He mentions that it is unknown whether or not the complementary summand is zero and in particular whether or not $\tilde{H}^{*}\left(\operatorname{Aut}\left(F_{\infty}\right) ; \mathbb{Q}\right)$ is always zero. In $[\mathbf{6}]$, Hatcher and Vogtmann again pose the question of whether or not the stable rational cohomology groups of $\operatorname{Aut}\left(F_{n}\right)$ and $\operatorname{Out}\left(F_{n}\right)$ all vanish, and show that it does

2000 Mathematics Subject Classification. Primary: 05C25, 20F32, 20J05; Secondary: 20F28, 55N91.

Key words. Graphs, free groups, moduli spaces, outer space, auter space. 
vanish in dimensions 1 through 6. A recent theorem of Madsen and Tillman gives (after inverting the prime 2) a product decomposition for the plus construction $B \Gamma^{+}$of the classifying space for stable mapping class groups; however, it is currently unknown to what extent this enables one to answer the question posed by Hatcher and Vogtmann.

Let $\hat{X}_{m}$ be the spine of outer space (see Culler and Vogtmann in [3]) and let $\hat{Q}_{m}=\hat{X}_{m} / \operatorname{Out}\left(F_{m}\right)$ be the corresponding moduli space of graphs. Similarly, let $X_{m}$ be the spine of auter space (see Hatcher and Vogtmann in [6]) and let $Q_{m}=X_{m} / \operatorname{Aut}\left(F_{m}\right)$ be the corresponding moduli space of pointed graphs. In this paper, we show that

Theorem 1. Let $i \in\{0,1\}$. For all positive integers $k$, either

$$
H^{4 k+i}\left(\operatorname{Out}\left(F_{\infty}\right) ; \mathbb{Q}\right) \neq 0
$$

or

$$
H^{4 k+i+1}\left(\hat{Q}_{m} ; \mathbb{Z}\right) \text { never stabilizes as } m \rightarrow \infty \text {. }
$$

Theorem 2. For all positive integers $k$, either

$$
H^{4 k}\left(\operatorname{Aut}\left(F_{\infty}\right) ; \mathbb{Q}\right) \neq 0
$$

or

$$
H^{4 k+1}\left(Q_{m} ; \mathbb{Z}\right) \text { never stabilizes as } m \rightarrow \infty \text {. }
$$

From calculations in [7] that

$$
H^{4}\left(\operatorname{Aut}\left(F_{\infty}\right) ; \mathbb{Q}\right)=H^{4}\left(\operatorname{Out}\left(F_{\infty}\right) ; \mathbb{Q}\right)=H^{5}\left(\operatorname{Aut}\left(F_{\infty}\right) ; \mathbb{Q}\right)=0,
$$

the above two theorems immediately show that

Corollary 3. The cohomology groups $H^{5}\left(\hat{Q}_{m} ; \mathbb{Z}\right)$ and $H^{6}\left(\hat{Q}_{m} ; \mathbb{Z}\right)$ never stabilize as $m \rightarrow \infty$.

Corollary 4. The cohomology group $H^{5}\left(Q_{m} ; \mathbb{Z}\right)$ never stabilizes as $m \rightarrow \infty$.

The two corollaries are true because as $m$ increases, torsion from increasingly higher primes is introduced in $H^{5}\left(\hat{Q}_{m} ; \mathbb{Z}\right), H^{6}\left(\hat{Q}_{m} ; \mathbb{Z}\right)$, and $H^{5}\left(Q_{m} ; \mathbb{Z}\right)$. There are natural inclusions $Q_{m} \longmapsto Q_{m+1}$, and it is known $[6]$ that the induced map $H^{i}\left(Q_{m+1} ; \mathbb{Q}\right) \rightarrow H^{i}\left(Q_{m} ; \mathbb{Q}\right)$ is an isomorphism for $m>3 i / 2$. It is therefore important to keep in mind that the above two corollaries only hold with respect to integral cohomology. 
A quick note about our notation is appropriate here. In general, groups without any additional structure will be written using multiplicative notation (e.g., $\mathbb{Z} / p \times \mathbb{Z} / p \cong(\mathbb{Z} / p)^{2}$ ) but modules like cohomology groups will be written using additive notation (e.g., $\mathbb{Z} / p \oplus \mathbb{Z} / p \cong 2(\mathbb{Z} / p)$ ).

In Section 2 we review the basics about outer and auter space, and in Section 3 we prove Theorem 1. Symmetry groups of graphs with $2 p-1$ holes are discussed in Section 4, which enables us to prove Theorem 2 in Section 5 .

This paper is based on a dissertation (see $[\mathbf{8}],[\mathbf{9}]$ ) written while the author was a student of Karen Vogtmann at Cornell, and the author would like to thank Prof. Vogtmann for her help and advice. The author would also like to thank Henry Glover for his helpful comments on this paper.

\section{Basics about spectral sequences and $\operatorname{Aut}\left(\boldsymbol{F}_{n}\right)$}

Let $G$ be a group acting cellularly on a finite dimensional CW-complex $X$ such that the $\operatorname{stabilizer} \operatorname{stab}_{G}(\delta)$ of every cell $\delta$ is finite and such that the quotient of $X$ by $G$ is finite. Further suppose that for every cell $\delta$ of $X$, the group $\operatorname{stab}_{G}(\delta)$ fixes $\delta$ pointwise. Let $M$ be a $G$-module. Recall (see [2]) that the equivariant cohomology groups of the $G$-complex $X$ with coefficients in $M$ are defined by

$$
H_{G}^{*}(X ; M)=H^{*}\left(G ; C^{*}(X ; M)\right)
$$

and that if in addition $X$ is contractible (which will usually, but not always, be the case in this paper) then

$$
H_{G}^{*}(X ; M)=H^{*}(G ; M) .
$$

In $[\mathbf{2}]$ a spectral sequence

$$
\tilde{E}_{1}^{r, s}=\prod_{[\delta] \in \Delta_{n}^{r}} H^{s}(\operatorname{stab}(\delta) ; M) \Rightarrow H_{G}^{r+s}(X ; M)
$$

is defined, where $[\delta]$ ranges over the set $\Delta_{n}^{r}$ of orbits of $r$-simplices $\delta$ in $X$.

If $M$ is $\mathbb{Z} / p$ or $\mathbb{Z}_{(p)}$ then a nice property should be noted about the spectral sequence (5). This property will greatly reduce the calculations we need to go through, and in general will make concrete computations possible. Since each group $\operatorname{stab}(\delta)$ is finite, a standard restrictiontransfer argument in group cohomology yields that $|\operatorname{stab}(\delta)|$ annihilates $H^{s}(\operatorname{stab}(\delta) ; M)$ for all $s>0$. (For examples of these sorts of arguments see [2].) Since all primes not equal to $p$ are divisible in $\mathbb{Z} / p$ or $\mathbb{Z}_{(p)}$, this in turn shows that the $p$-part of $|\operatorname{stab}(\delta)|$ annihilates $H^{s}(\operatorname{stab}(\delta) ; M)$ 
for $s>0$. In particular, if $p$ does not divide some $|\operatorname{stab}(\delta)|$, then this $[\delta]$ does not contribute anything to the spectral sequence (5) except in the horizontal row $s=0$. It follows that if our coefficients are $\mathbb{Z} / p$ or $\mathbb{Z}_{(p)}$ then we are mainly just concerned with the simplices $\delta$ which have "p-symmetry".

We now specialize to the cases where $G$ is $\operatorname{Out}\left(F_{n}\right)$ or $\operatorname{Aut}\left(F_{n}\right)$ and $X$ is either the spine $\hat{X}_{n}$ of "outer space" or the spine $X_{n}$ of "auter space". Hatcher and Vogtmann's definition of auter space closely follows Culler and Vogtmann's (prior) definition of outer space, except that the graphs arising have basepoints. We review some basic properties and definitions of auter space below, where we concentrate on auter space because that is where most of the calculations in this paper will take place. Most of these facts can be found in $[\mathbf{3}],[\mathbf{6}],[\mathbf{1 2}]$, and $[\mathbf{1 3}]$.

Consider the automorphism group $\operatorname{Aut}\left(F_{n}\right)$ of a free group $F_{n}$ of rank $n$ (where $n$ will be $2 p-1$ for most of our work). Let $\left(R_{n}, v_{0}\right)$ be the $n$-leafed rose, a wedge of $n$ circles. We say a basepointed graph $\left(G, x_{0}\right)$ is admissible if it has no free edges, all vertices except the basepoint have valence at least three, and there is a basepoint-preserving continuous map $\phi: R_{n} \rightarrow G$ which induces an isomorphism on $\pi_{1}$. The triple $\left(\phi, G, x_{0}\right)$ is called a marked graph. Two marked graphs $\left(\phi_{i}, G_{i}, x_{i}\right)$ for $i=0,1$ are equivalent if there is a homeomorphism $\alpha:\left(G_{0}, x_{0}\right) \rightarrow$ $\left(G_{1}, x_{1}\right)$ such that $\left(\alpha \circ \phi_{0}\right)_{\#}=\left(\phi_{1}\right)_{\#}: \pi_{1}\left(R_{n}, v_{0}\right) \rightarrow \pi_{1}\left(G_{1}, x_{1}\right)$. Define a partial order on the set of all equivalence classes of marked graphs by setting $\left(\phi_{0}, G_{0}, x_{0}\right) \leq\left(\phi_{1}, G_{1}, x_{1}\right)$ if $G_{1}$ contains a forest (a disjoint union of trees in $G_{1}$ which contains all of the vertices of $G_{1}$ ) such that collapsing each tree in the forest to a point yields $G_{0}$, where the collapse is compatible with the maps $\phi_{0}$ and $\phi_{1}$.

From [5] and [6] we have that $\operatorname{Aut}\left(F_{n}\right)$ acts with finite stabilizers on a contractible space $X_{n}$. The space $X_{n}$ is the geometric realization of the poset of marked graphs that we defined above. Let $Q_{n}$ be the quotient of $X_{n}$ by $\operatorname{Aut}\left(F_{n}\right)$. Note that the CW-complex $Q_{n}$ is not necessarily a simplicial complex. Since $\operatorname{Aut}\left(F_{n}\right)$ has a torsion free subgroup of finite index [5] and it acts on the contractible, finite dimensional space $X_{n}$ with finite stabilizers and finite quotient, $\operatorname{Aut}\left(F_{n}\right)$ has finite vcd.

Let $p$ be an odd prime number, and let $\mathbb{Z}_{(p)}$ be the localization of $\mathbb{Z}$ at the prime ideal $(p)$. Then we can apply the spectral sequence (5) to get

$$
\tilde{E}_{1}^{r, s}=\prod_{[\delta] \in \Delta_{n}^{r}} H^{s}\left(\operatorname{stab}(\delta) ; \mathbb{Z}_{(p)}\right) \Rightarrow H^{r+s}\left(\operatorname{Aut}\left(F_{n}\right) ; \mathbb{Z}_{(p)}\right)
$$

where $[\delta]$ ranges over the set $\Delta_{n}^{r}$ of orbits of $r$-simplices $\delta$ in $X_{n}$. 
The spectral sequence $(6)$ requires as input the stabilizers $\operatorname{stab}_{\mathrm{Aut}\left(F_{n}\right)}(\delta)$ of simplices $\delta$ in $X_{n}$. Smillie and Vogtmann [12] examined the structure of these stabilizers in detail, and we list their results here. Consider a given $r$-simplex

$$
\left(\phi_{r}, G_{r}, x_{r}\right)>\cdots>\left(\phi_{1}, G_{1}, x_{1}\right)>\left(\phi_{0}, G_{0}, x_{0}\right)
$$

with corresponding forest collapses

$$
\left(H_{r} \subseteq G_{r}\right), \ldots,\left(H_{2} \subseteq G_{2}\right),\left(H_{1} \subseteq G_{1}\right) .
$$

For each $i \in 0,1, \ldots, r$, let $F_{i}$ be the inverse image under the map

$$
G_{r} \rightarrow \cdots \rightarrow G_{i+1} \rightarrow G_{i}
$$

of forest collapses, of the forest $H_{i}$. That is, we have

$$
F_{r} \subseteq \cdots \subseteq F_{2} \subseteq F_{1} \subseteq G_{r}
$$

It is shown in [12] that the stabilizer of the simplex under consideration is isomorphic to the group $\operatorname{Aut}\left(G_{r}, F_{1}, \ldots, F_{r}, x_{r}\right)$ of basepointed automorphisms of the graph $G_{r}$ that respect each of the forests $F_{i}$. For example, the stabilizer of a point $\left(\phi, G, x_{0}\right)$ in $X_{n}$ is isomorphic to $\operatorname{Aut}\left(G, x_{0}\right)$.

\section{Graphs without basepoints}

Theorem 1 is a direct consequence of the stability theorems in [5] and the spectral sequence calculations in [4].

Proof of Theorem 1: From [5],

$$
H^{4 k+i}\left(\operatorname{Out}\left(F_{\infty}\right) ; \mathbb{Q}\right)=H^{4 k+i}\left(\operatorname{Aut}\left(F_{\infty}\right) ; \mathbb{Q}\right)
$$

and if $m \geq 4 k^{2}+10 k+1+i^{2} / 4+2 i k+5 i / 2$, then the standard map

$$
H^{4 k+i}\left(\operatorname{Aut}\left(F_{m}\right) ; \mathbb{Z}\right) \rightarrow H^{4 k+i}\left(\operatorname{Out}\left(F_{m}\right) ; \mathbb{Z}\right)
$$

is an isomorphism. Observe that $H^{4 k+i}\left(\operatorname{Out}\left(F_{4 k^{2}+10 k+1+i^{2} / 4+2 i k+5 i / 2}\right)\right.$; $\mathbb{Z})=H^{4 k+i}\left(\operatorname{Out}\left(F_{\infty}\right) ; \mathbb{Z}\right)$ is a finitely generated abelian group. If it contains a torsion free summand isomorphic to $\mathbb{Z}$, then we are done and $H^{4 k+i}\left(\operatorname{Out}\left(F_{\infty}\right) ; \mathbb{Q}\right) \neq 0$. Otherwise, choose a prime $q$ such that $q+1 \geq 4 k^{2}+10 k+1+i^{2} / 4+2 i k+5 i / 2$ and so that for all primes $p \geq q$ there is no $p$-torsion in $H^{4 k+i}\left(\operatorname{Out}\left(F_{\infty}\right) ; \mathbb{Z}\right)$. We will show that $H^{4 k+i+1}\left(\hat{Q}_{p+1} ; \mathbb{Z}\right)$ has $p$-torsion for infinitely many primes $p$, which will prove the theorem. 
Let $p \geq \max \{q, 25\}$ with $p \equiv 3(\bmod 4)$. (Note that there are infinitely many possibilities for $p$, as there are infinitely many primes that are greater than a given number and congruent to 3 modulo 4.) Because $H^{4 k+i}\left(\operatorname{Out}\left(F_{p+1}\right) ; \mathbb{Z}\right)$ has no $p$-torsion, there is also no $p$-torsion in $H^{4 k+i}\left(\operatorname{Out}\left(F_{p+1}\right) ; \mathbb{Z}_{(p)}\right)$. From the calculation of Glover and Mislin in [4] of the $E_{2}$-page of the equivariant spectral sequence used to calculate $H^{*}\left(\operatorname{Out}\left(F_{p+1}\right) ; \mathbb{Z}_{(p)}\right)$, we know that this $E_{2}$-page, in the rows $0 \leq s<2(p-1)$, is given by

$$
E_{2}^{r, s}= \begin{cases}H^{r}\left(\hat{Q}_{p+1} ; \mathbb{Z}_{(p)}\right) & s=0 \\ \mathbb{Z} / p & r=0 \text { and } s=4 k>0, k \in \mathbb{Z}^{+} \\ \left(n_{p}\right) \mathbb{Z} / p & r=1 \text { and } s=4 k>0, k \in \mathbb{Z}^{+} \\ 0 & \text { otherwise }\end{cases}
$$

where $n_{p}=(p-1) / 12-\epsilon_{p}$ and $\epsilon_{p} \in\{0,1\}$. Since $p \geq 25$, note that $n_{p} \geq 1$.

Hence a class $\hat{\alpha} \in E_{2}^{i, 4 k}$ in the $E_{2}$-page survives at least until the $E_{4 k+1}$-page. The class $\hat{\alpha} \in E_{2}^{i, 4 k}$ cannot survive to the $E_{\infty}$ page, however, because there is no $p$-torsion in the finite (since $H^{4 k+i}\left(\operatorname{Out}\left(F_{p+1}\right)\right.$; $\mathbb{Q})=0)$ additive group $H^{4 k+i}\left(\operatorname{Out}\left(F_{p+1}\right) ; \mathbb{Z}_{(p)}\right)$.

It follows that there is $p$-torsion in

$$
E_{4 k+1}^{4 k+i+1,0}=H^{4 k+i+1}\left(\hat{Q}_{p+1} ; \mathbb{Z}_{(p)}\right)
$$

Thus $H^{4 k+i+1}\left(\hat{Q}_{p+1} ; \mathbb{Z}\right)$ has $p$-torsion.

\section{Symmetry groups of graphs}

We will use spectral sequence (6) to compute a portion of the cohomology of $\operatorname{Aut}\left(F_{n}\right)$. Since our coefficient ring is $\mathbb{Z}_{(p)}$, we have already remarked that for the terms in the spectral sequence above the horizontal axis, we are concerned only with simplices whose stabilizers are divisible by $p$. In addition, the stabilizer of a simplex consists of graph automorphisms that respect the forest collapses in the simplex. We will find which simplices arise in the case $n=2 p-1$. In other words, we want to calculate which graphs $G$ with a $\mathbb{Z} / p$ action on them have $\pi_{1}(G) \cong F_{n}$. Recall that a $\mathbb{Z} / p$-graph $G$ is reduced if it contains no $\mathbb{Z} / p$-invariant subforests. 
We now examine the cohomology of the quotient $Q_{n}$ of the spine $X_{n}$ of auter space. There are natural inclusions $Q_{m} \longmapsto Q_{m+1}$, and it is known $[6]$ that the induced map $H^{i}\left(Q_{m+1} ; \mathbb{Q}\right) \rightarrow H^{i}\left(Q_{m} ; \mathbb{Q}\right)$ is an isomorphism for $m>3 i / 2$. Our goal is to show that, in contrast, $H^{5}\left(Q_{m} ; \mathbb{Z}\right)$ never stabilizes as $m \rightarrow \infty$. This is done by showing that as $m$ increases, torsion from increasingly higher primes is introduced in $H^{5}\left(Q_{m} ; \mathbb{Z}\right)$. To this end, we do specific calculations in the spectral sequence (5) applied to the action of $\operatorname{Aut}\left(F_{n}\right)$ on $X_{n}$ for $n=2 p-1$. The $E_{2}^{r, 0}$-term of this spectral sequence is $H^{r}\left(Q_{n} ; \mathbb{Z}_{(p)}\right)$, and the sequence converges to $H^{r}\left(\operatorname{Aut}\left(F_{n}\right) ; \mathbb{Z}_{(p)}\right)$. Results from Hatcher and Vogtmann [6] on the cohomology of $\operatorname{Aut}\left(F_{n}\right)$ are then used to obtain the result.

In this section, we do the ground work necessary to compute the $E_{1}$-page of the spectral sequence: we find all simplices of $X_{n}$ with $p$-symmetry and compute the cohomology of the stabilizers of these simplices with coefficients in $\mathbb{Z}_{(p)}$. In Section 5 we will compute the $E_{2}$-page of the spectral sequence, and use this calculation to obtain the result.

Unless otherwise stated, $p \geq 5$ will be prime and $n=2 p-1$. The assumption that $p \geq 5$ is for convenience more than any other reason, as the main results will only consider arbitrarily large primes $p$ and so we should not devote extra time to the (fairly easy to resolve) complications introduced by considering the prime $p=3$. These complications arise from the fact that the dihedral group $D_{6}$ is the same as the symmetric group $S_{3}$, so that we cannot distinguish between dihedral and symmetric symmetry in that case.

We now define some graphs that we will need for this section. (Refer to Figures 1 and 2 for illustrations of most of these graphs.) Let $\Theta_{p-1}$ be the graph with two vertices and $p$ edges, each of which goes from one vertex to the other (see Figure 1). Say the "leftmost vertex" of $\Theta_{p-1}$ is the basepoint. Hence when we write $\Theta_{p-1} \vee R_{p-1}$ then we are stipulating that the rose $R_{p-1}$ is attached to the non-basepointed vertex of $\Theta_{p-1}$, while when we write $R_{p-1} \vee \Theta_{p-1}$ then we are saying that the rose is attached to the basepoint of $\Theta_{p-1}$. Let $\Phi_{2(p-1)}$ be a graph with $3 p$ edges $a_{1}, \ldots, a_{p}, b_{1}, \ldots, b_{p}, c_{1}, \ldots, c_{p}$, and $p+3$ vertices $v_{1}, \ldots, v_{p}, x, y, z$. The basepoint is $x$ and each of the edges $a_{i}$ begin at $x$ and end at $v_{i}$. The edges $b_{i}$ and $c_{i}$ begin at $y$ and $z$, respectively, and end at $v_{i}$. Note that there are obvious actions of $\mathbb{Z} / p$ on $\Theta_{p-1}$ and $\Phi_{2(p-1)}$, given by rotation, and that these actions are unique up to conjugacy. Let $\Psi_{2(p-1)}$ be the graph obtained from $\Phi_{2(p-1)}$ by collapsing all of the edges $a_{i}$ to a point. 
Let $\Omega_{2(p-1)}$ be the graph obtained from $\Phi_{2(p-1)}$ by collapsing either the edges $b_{i}$ or the edges $c_{i}$ (the resulting graphs are isomorphic) to a point. Note that the only difference between $\Psi_{2(p-1)}$ and $\Omega_{2(p-1)}$ is where the basepoint is located.
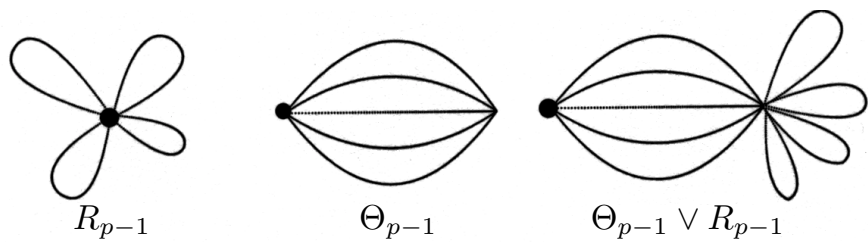

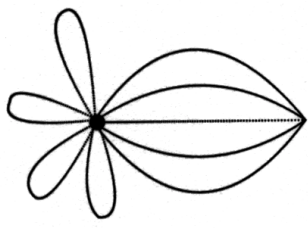

$R_{p-1} \vee \Theta_{p-1}$

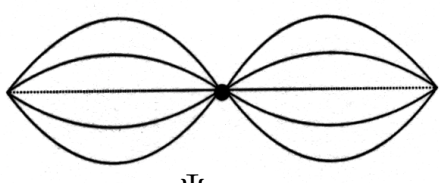

$\Psi_{2(p-1)}$

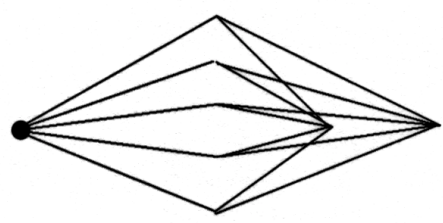

$\Phi_{2(p-1)}$

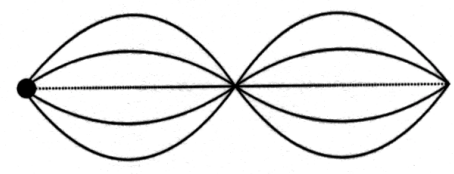

$\Omega_{2(p-1)}$

FIGURE 1. Some graphs with $p$-symmetry

Given a finite subgroup $G$ of $\operatorname{Aut}\left(F_{n}\right)$ for some integer $n$, we say that a marked graph

$$
\eta^{1}: R_{r} \rightarrow \Gamma^{1}
$$

is a $G$-equivariant blowup in the fixed point space $X_{r}^{G}$ of a marked graph

$$
\eta^{2}: R_{r} \rightarrow \Gamma^{2}
$$

if there is a 1-simplex $\eta^{1}>\eta^{2}$ in $X_{r}^{G}$. 

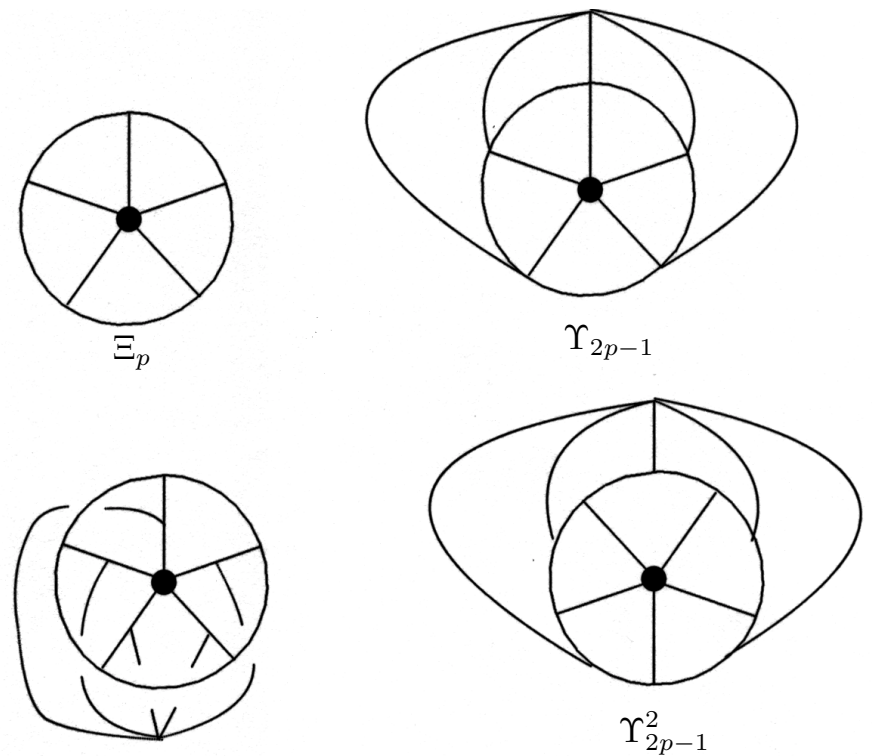

$\Upsilon_{2 p-1}^{1}$

Figure 2. Some graphs with $D_{2 p}$-symmetry

Let $\Upsilon_{2 p-1}^{1}$ and $\Upsilon_{2 p-1}^{2}$ be the two possible graphs that can be obtained from $\Upsilon_{2 p-1}$ by equivariantly blowing up the $p$ valence 4 vertices into $2 p$ valence 3 vertices. That is, $\Upsilon_{2 p-1}^{1}$ can be obtained by first taking a $p$-gon and then attaching $p$ free edges to the $p$ vertices of the $p$-gon. Say each of these new edges $e_{i}$ begins at the vertex $x_{i}$ and ends at the vertex $y_{i}$, and suppose that the vertices $x_{i}$ are the ones that are attached to the $p$-gon. Now form the 1-skeleton of the double cone or suspension over the $p$ vertices $y_{i}$. This gives the graph $\Upsilon_{2 p-1}^{1}$. The graph $\Upsilon_{2 p-1}^{2}$ can be thought of as follows: First take a $p$-gon and cone off over the $p$ vertices of the $p$-gon. Now also cone off over the $p$ midpoints of the $p$ edges of the $p$-gon. Note that there is an obvious $\mathbb{Z}_{p}$-action on each of $\Theta_{p-1}, \Xi_{p}, \Upsilon_{2 p-1}, \Upsilon_{2 p-1}^{1}$, and $\Upsilon_{2 p-1}^{2}$.

Let $\Xi_{p}$ be the 1 -skeleton of the cone over a $p$-gon, so that $\Xi_{p}$ has $p+1$ vertices and $2 p$ edges, one vertex has valence $p$ and the other $p$ vertices all have valence 3. Let $\Upsilon_{2 p-1}$ be the 1-skeleton of the suspension of a $p$-gon. Hence $\Upsilon_{2 p-1}$ has $p+2$ vertices and $3 p$ edges; two of the vertices have valence $p$ and the other $p$ have valence 4 . 

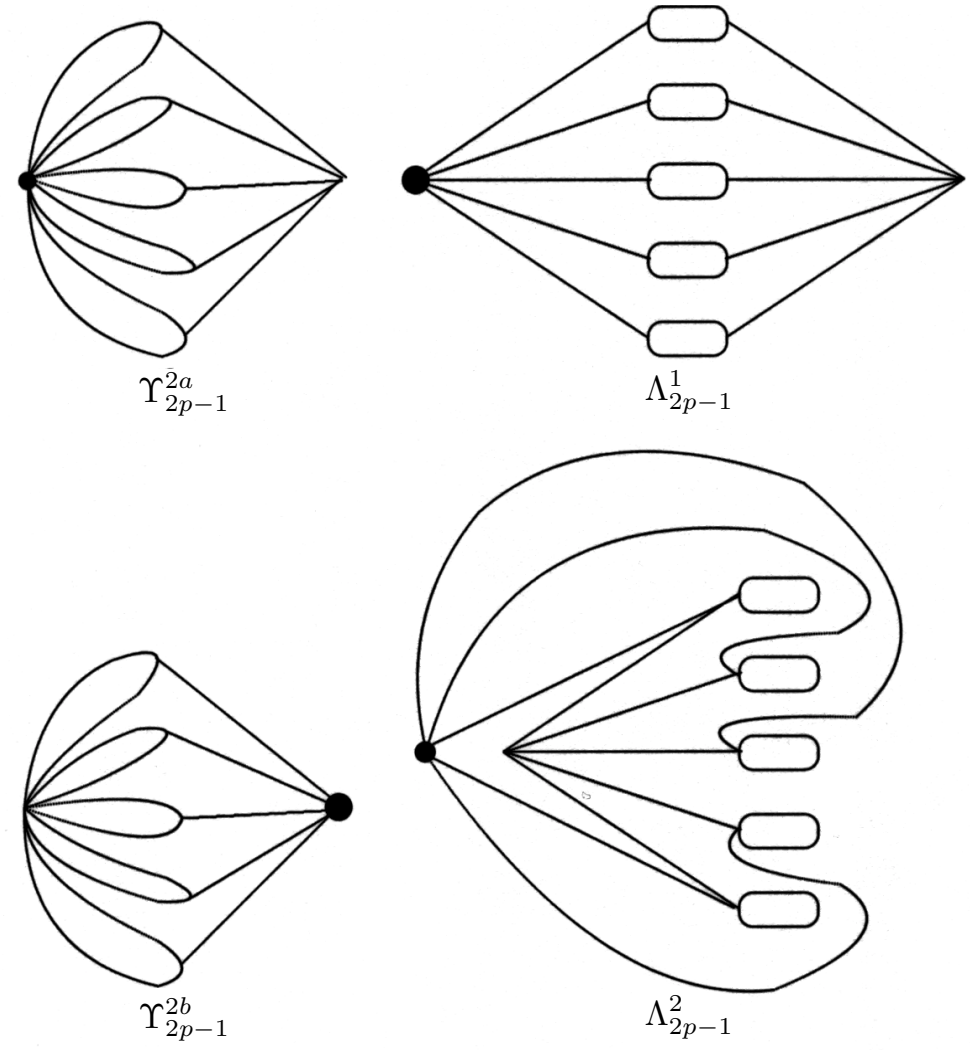

FIGURE 3. Some graphs with (cohomologically) $\Sigma_{p}$-symmetry

Choose basepoints for the graphs $\Theta_{p-1}, \Upsilon_{2 p-1}, \Upsilon_{2 p-1}^{1}$, and $\Upsilon_{2 p-1}^{2}$ as illustrated in Figure 2: Let the vertex on the "leftmost" side of $\Theta_{p-1}$ be the basepoint. Additionally, orient $\Upsilon_{2 p-1}, \Upsilon_{2 p-1}^{1}$, and $\Upsilon_{2 p-1}^{2}$ so that one of their valence $p$ vertices is on the "left" and the other is on the "right" and choose the leftmost vertex to be the basepoint. Writing $R_{p} \vee \Theta_{p-1}$ will mean that the two graphs are wedged together at the basepoint of $\Theta_{p-1}$, while writing $\Theta_{p-1} \vee R_{p}$ will mean that the non-basepointed vertex of $\Theta_{p-1}$ is wedged to the vertex of $R_{p}$. Let $\Upsilon_{2 p-1}^{2 a}$ be the graph obtained from $\Upsilon_{2 p-1}^{2}$ by collapsing the leftmost $p$ edges and let $\Upsilon_{2 p-1}^{2 b}$ be the one obtained by collapsing the rightmost $p$ edges. Refer to Figure 3 for pictures of these graphs. Figure 3 also depicts two graphs $\Lambda_{2 p-1}^{1}$ and $\Lambda_{2 p-1}^{2}$ which will be used in the proof of Lemma 7 . 
For the next lemma (Lemma 7) only, we will consider the above graphs not to have basepoints specified. The basepoint is just assumed to be located at some spot which is invariant under the $\mathbb{Z} / p$ action. (This assumption is for convenience rather than anything else, so that we will not need to introduce several separate subcases, each corresponding to a different location for the basepoint.)

We will be looking at the standard equivariant spectral sequence (5) applied to calculating the cohomology groups $H^{*}\left(\operatorname{Aut}\left(F_{n}\right) ; \mathbb{Z}_{(p)}\right)$. In particular, we will be looking at the $E_{1}$ page of this spectral sequence only in rows 0 through $2 p-3$, and often just in rows 1 though $2 p-3$. One interesting fact about these rows is that they allow us to distinguish between simplices that have stabilizers whose cohomology is $\mathbb{Z} / p, D_{2 p}$, or $\Sigma_{p}$. It is well known that

$$
\begin{aligned}
H^{*}\left(\mathbb{Z} / p ; \mathbb{Z}_{(p)}\right) & =\mathbb{Z}_{(p)}\left[x_{2}\right] /\left(p x_{2}\right), \\
H^{*}\left(D_{2 p} ; \mathbb{Z}_{(p)}\right) & =\mathbb{Z}_{(p)}\left[x_{4}\right] /\left(p x_{4}\right), \\
\text { and } \quad H^{*}\left(\Sigma_{p} ; \mathbb{Z}_{(p)}\right) & =\mathbb{Z}_{(p)}\left[x_{2(p-1)}\right] /\left(p x_{2(p-1)}\right),
\end{aligned}
$$

where $x_{2}, x_{4}$ and $x_{2(p-1)}$ are generators of dimensions 2,4 and $2(p-1)$, respectively. Hence if a simplex of $X_{n}$ has stabilizer isomorphic to $\mathbb{Z} / p$ or $D_{2 p}$ then it will contribute something to the $E_{1}$ page of the spectral sequence in some of the rows 1 through $2 p-3$. On the other hand, if its stabilizer is isomorphic to $\Sigma_{p}$, then it will not contribute anything to the $E_{1}$ page of the spectral sequence in the given rows.

Define an $r$-simplex in the $p$-singular locus of $X_{n}$ to have exactly $\mathbb{Z} / p$ symmetry if it contributes exactly one copy of $\mathbb{Z} / p$ to each of the entries $E_{1}^{r, 2 k}, 0<2 k<2(p-1)$, in the $E_{1}$ page of the spectral sequence. Define an $r$-simplex in the $p$-singular locus of $X_{n}$ to have at most dihedral symmetry if it contributes exactly one copy of $\mathbb{Z} / p$ to each of the entries $E_{1}^{r, 4 k}, 0<4 k<2(p-1)$, in the $E_{1}$ page of the spectral sequence.

The next lemma examines which vertices in $X_{n}$ contribute to the spectral sequence in the given rows. The proof of the lemma actually explicitly enumerates which graphs have $p$-symmetry, which will be very useful to us later.

Lemma 7. Let $p \geq 5$ be prime and set $n=2 p-1$. Let the marked graph $\left(\xi, G, x_{0}\right)$ be a vertex in the p-singular locus of $X_{n}$. Then the cohomology $H^{*}\left(\operatorname{Aut}\left(G, x_{0}\right) ; \mathbb{Z}_{(p)}\right)$ of the stabilizer of this vertex is the same as the cohomology with $\mathbb{Z}_{(p)}$ coefficients of one of $D_{2 p}, D_{2 p} \times \Sigma_{p}$, $\Sigma_{p}, \Sigma_{p} \times \Sigma_{p},\left(\Sigma_{p} \times \Sigma_{p}\right) \rtimes \mathbb{Z} / 2$ or $\Sigma_{2 p}$ 
Proof: From [1] and [11], we see that $p^{2}$ is an upper bound for the order of any $p$-subgroup of $\operatorname{Aut}\left(F_{n}\right)$. Thus $p^{2}$ is an upper bound for the order of a maximal $p$-subgroup $P$ of $\operatorname{Aut}\left(G, x_{0}\right)$. Since all possible choices for $P$ are abelian (i.e., there are only three possibilities: $\mathbb{Z} / p, \mathbb{Z} / p^{2}$, and $\mathbb{Z} / p \times \mathbb{Z} / p$ ), we can apply Swan's theorem (see [14]) to see that

$$
H^{*}\left(\operatorname{Aut}\left(G, x_{0}\right) ; \mathbb{Z}_{(p)}\right)=H^{*}\left(P ; \mathbb{Z}_{(p)}\right)^{N_{\mathrm{Aut}\left(G, x_{0}\right)}(P)} .
$$

We now look at each of the individual cases $P=\mathbb{Z} / p, \mathbb{Z} / p^{2}$, and $\mathbb{Z} / p \times \mathbb{Z} / p$.

Case 1: We will first examine the case where $P=\mathbb{Z} / p^{2}$. In this case, we have that $p^{2}$ edges $e_{1}, \ldots, e_{p^{2}}$ in $G$ are rotated around by $P$. An examination of all possible ways that these edges could be connected together, keeping in mind that $G$ is admissible, reveals that this case is impossible. For example, the first subcase is that all of the $e_{i}$ begin and end at the same vertex. This is not possible because the fundamental group of $R_{p^{2}}$ is too large for it to be a subgraph of $G$. For the next subcase, suppose each edge goes from some vertex $y_{1}$ to some other vertex $y_{2}$. Then they form a $\Theta_{p^{2}-1}$ inside $G$, which is also impossible. In the next subcase, the edges begin at one common vertex $y_{0}$ and end at $p^{2}$ distinct vertices $y_{1}, \ldots, y_{p^{2}}$. Since the graph $G$ is admissible, it has no free edges and every nonbasepointed vertex has valence at least 3 . So all of the vertices $y_{1}, \ldots, y_{p^{2}}$ have to connect up in some manner, and in doing so they will violate the fact that $\pi_{1}(G)=F_{2 p-1}$. The final three subcases, in which the $e_{i}$ either form a $p^{2}$-gon, have no common vertices, or form loops with $p^{2}$ distinct endpoints, are similar. Hence $P$ will never be $\mathbb{Z} / p^{2}$ and this case will not occur.

Case 2: Next we will examine the case where $P=\mathbb{Z} / p \times \mathbb{Z} / p=(\alpha) \times(\beta)$. The first cyclic summand must rotate $p$ edges $e_{1}, e_{2}, \ldots, e_{p}$ of $\Gamma$. Without loss of generality, we may assume that the basepoint $*$ is one of the endpoints of each $e_{i}$. Now if $\beta$ sends all of the $e_{i}$ to another whole collection $\beta e_{i}$ (with $\left\{e_{i}\right\}$ disjoint from $\left\{\beta e_{j}\right\}$ ) then the basepoint $*$ must be one of the endpoints of each $\beta^{j} e_{i}$ also; therefore, we obtain at least $p^{2}$ edges emanating from the basepoint $*$ which are moved by $\alpha$ and $\beta$. This implies that the rank of $\pi_{1}(\Gamma)$ is at least $p(p-1)$ (i.e., the best that can happen is that $p$ copies of $\Theta_{p-1}$ are wedged together at the basepoint), which is too large as $p \geq 5$.

So $\beta$ does not send the $e_{i}$ to another whole collection $\beta e_{i}$ of edges disjoint from the $e_{i}$. Without loss of generality, $(\beta)$ fixes the edges $e_{i}$ (by replacing $\beta$ by $\beta-\alpha^{j}$ if necessary). Hence the collection $\left\{e_{i}\right\}$ is $P$-invariant. Now $\beta$ must rotate $p$ other edges $f_{1}, f_{2}, \ldots, f_{p}$. The $e_{i}$ do 
not form a $p$-gon as $*$ is an endpoint of each of them. Hence the $e_{i}$ form either a rose, a star, or a $\Theta$-graph. If they form a rose or a star, then the $f_{i}$ must form a $\Theta_{p-1}$ else the rank of $\pi_{1}(\Gamma)$ is larger than $2 p-1$. If the $e_{i}$ form a $\Theta$-graph $\Theta_{p-1}$, then there are $p$ holes available in the rank of $\pi_{1}(\Gamma)$ for the other edges of $\Gamma$ to use up.

By doing the sort of case-by-case analysis that we did in the previous paragraph, we see that $G$ must be one of the following graphs (listed in increasing order with respect to the number of vertices):

- $R_{p} \vee \Theta_{p-1}$, whose automorphism group has the same cohomology as $\Sigma_{p} \times \Sigma_{p}$

- $\Theta_{2 p-1}$, whose automorphism group is $\Sigma_{2 p}$.

- $\Theta_{p-1} \vee \Theta_{p-1}$, plus one additional edge $e$ attached in some manner to the existing vertices. The automorphism group here will have the same cohomology as either $\Sigma_{p} \times \Sigma_{p}$ or $\left(\Sigma_{p} \times \Sigma_{p}\right) \rtimes \mathbb{Z} / 2$.

- $\Theta_{p-1} \coprod \Theta_{p-1}$, with one additional edge $e_{1}$ attached going from an already existing vertex of one of the $\Theta$-graphs to one on the other $\Theta$-graph, after which we sequentially attach another edge $e_{2}$ to that resulting graph. The endpoints of $e_{2}$ can be attached to any of the already existing vertices, or they can be attached anywhere in the interior of $e_{1}$. The automorphism group here will have the same cohomology as either $\Sigma_{p} \times \Sigma_{p}$ or $\left(\Sigma_{p} \times \Sigma_{p}\right) \rtimes \mathbb{Z} / 2$.

- $\Theta_{p-1} \vee \Xi_{p}$, with automorphism group $\Sigma_{p} \times D_{2 p}$.

Case 3: For the final case, $P=\mathbb{Z} / p=(\alpha)$. We want to show that all of the $p$-symmetries in the graph $G$ are also at least $D_{2 p}$-symmetries. That is, in addition to the rotation by $\mathbb{Z} / p$, there is also a dihedral "flip". We will be able to get this result because $n=2 p-1$ is not large enough with respect to $p$ for us to be able to generate graphs $G$ with $\pi_{1}(G)=F_{n}$ that have $\mathbb{Z} / p$-symmetries but not $D_{2 p}$-symmetries.

We have $P=\mathbb{Z} / p$ acting on a graph whose fundamental group has rank $n=2 p-1$. As before, there exist at least $p$ edges $e_{1}, \ldots, e_{p}$ that $P$ rotates. If these edges form a $\Theta_{p-1}$ or an $R_{p}$, we are done. This is because now $P$ cannot move any other edges of $G$, else we are in the case of the previous paragraph where $P=\mathbb{Z} / p \times \mathbb{Z} / p$. As the automorphism groups of both $\Theta_{p-1}$ and $R_{p}$ contain the symmetric group $\Sigma_{p}$, we are done in this subcase.

Now suppose we are in the other extreme subcase, the one where $e_{1}, \ldots, e_{p}$ have no endpoints in common. Choose a minimal path $\gamma_{1}$ 
from $e_{1}$ to the basepoint. Since $P=(\alpha)$ fixes the basepoint, we have that $\gamma_{i}:=\alpha^{i-1} \gamma_{1}$ is a minimal path from $e_{i}$ to $x_{0}$ for all $i=1, \ldots, p$. Since none of the endpoints of the $e_{i}$ can be the basepoint, there are at least $p$ distinct edges $f_{1}, \ldots, f_{p}$ in $\gamma_{1}, \ldots, \gamma_{p}$, respectively. We can also assume that each $f_{i}$ has at least one endpoint that is not the basepoint. Because $G$ has no free edges, no separating edges, and all non-basepointed vertices have valence at least three, another case-by-case analysis reveals that since $\pi_{1}(G)$ must have rank less than $2 p$, the graph is forced to be either $\Upsilon_{2 p-1}^{1}, \Upsilon_{2 p-1}^{2}$, or $\Lambda_{2 p-1}^{1}$. The first two of these graphs have dihedral symmetry, while the last has automorphism group with the same cohomology as $\Sigma_{p}$.

The next case is the one in which the $e_{i}$ are all loops with $p$ distinct endpoints $y_{i}$. As in the previous case, we can choose a $\mathbb{Z} / p$ equivariant path from each $y_{i}$ to the basepoint. The admissibility conditions on the graph only allow one possibility, namely the graph $\Lambda_{2 p-1}^{2}$. As this graph has automorphism group with the same cohomology as $\Sigma_{p}$, we are finished with this case.

Next consider the case where the $e_{i}$ form a $p$-gon. Since the vertices of $G$ have valence at least 3 , there must be $p$ other edges $f_{1}, \ldots, f_{p}$ in $G$ that each start at one of the $p$ vertices of the $p$-gon. Since $G$ is admissible and the rank of $\pi_{1}(G)$ is $2 p-1$, these additional edges cannot also join up to form a $p$-gon. (Why? Both $p$-gons still need to connect up to the basepoint in some way, and in connecting up to $x_{0}$ the rank of the fundamental group of $G$ will be forced too high.) In addition, the edges must have some vertices in common, else we reduce to the previous case; therefore, the $f_{i}$ are all forced to end at some common vertex $y_{0}$. In other words, we have a $\Xi_{p}$ embedded in $G$. If $P$ doesn't move any other edges in $G$, we are done since $\Xi_{p}$ has dihedral symmetry. If some other edges $g_{1}, \ldots, g_{p}$ are moved, they must also be attached to the $p$-gon that the $e_{i}$ form, or we will have two independent $\mathbb{Z} / p$-actions and be in the case $P=\mathbb{Z} / p \times \mathbb{Z} / p$. None of the following cases can happen, else $\operatorname{rank}\left(\pi_{1}(G)\right) \geq 2 p$ :

- The other endpoints of the $g_{i}$ all connect to $y_{0}$.

- The other endpoints of the $g_{i}$ also connect to the $p$-gon formed by the $e_{i}$.

- The other endpoints of the $g_{i}$ form $p$ other distinct vertices.

Hence these other endpoints all have to connect to some other common vertex $y_{1}$, forming another copy of $\Xi_{p}$ in $G$. Thus $G$ must be the graph $\Upsilon_{2 p-1}$, which certainly has dihedral symmetry. 


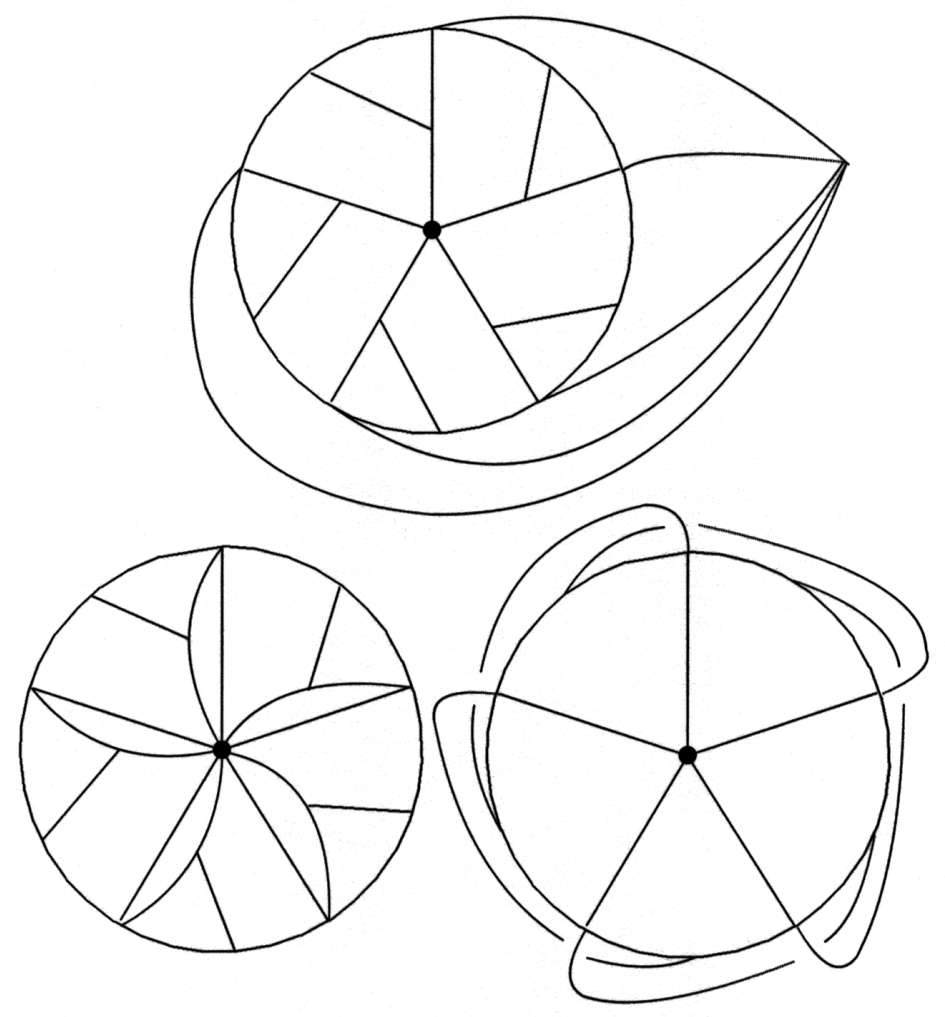

FiguRE 4 . Graphs whose symmetry groups are exactly $\mathbb{Z} / p$

For the final case, the edges $e_{i}$ have one common vertex $y_{0}$, and end in $p$ other distinct vertices $y_{1}, \ldots, y_{p}$. In addition, i) there are no $p$-gons in $G$, ii) there are no collections of $p$ edges in $G$ that are rotated by $P$ and that have no common vertices, and iii) there are no collections of $p$ edges in $G$ that are rotated by $P$ and that each form loops with distinct endpoints. Since all of $y_{1}, \ldots, y_{p}$ have valence three, $P$ must rotate two other collections of edges $\left\{f_{1}, \ldots, f_{p}\right\}$ and $\left\{g_{1}, \ldots, g_{p}\right\}$ that begin at the vertices $y_{1}, \ldots, y_{p}$ and end at the vertices $z_{1}$ and $z_{2}$, respectively. Note that since $\pi_{1}(G)=F_{2 p-1},\left|\left\{y_{0}, z_{1}, z_{2}\right\}\right| \geq 2$. Also note that $P$ cannot move any other edges of $G$ except the ones we have listed. In this case, 
the symmetric group $\Sigma_{p}$ acts on the collections of edges defined above, and so the cohomology of the group of graph automorphisms of the graph is the same as that of the symmetric group. If $\left|\left\{y_{0}, z_{1}, z_{2}\right\}\right|=2$ then the only edges in the graph are the $e_{i}, f_{i}$, and $g_{i}$ and the graph is either $\Upsilon_{2 p-1}^{2 a}$ or $\Upsilon_{2 p-1}^{2 b}$. On the other hand, if $\left|\left\{y_{0}, z_{1}, z_{2}\right\}\right|=3$, then the graph has one additional edge besides the $e_{i}, f_{i}$, or $g_{i}$. Accordingly, the graph looks like a $\Phi_{2 p-1}$ (see Figure 1$)$ with one additional edge added. This additional edge can go from any of the $\left\{y_{0}, z_{1}, z_{2}\right\}$ to any other one, including possibly the same one. In any case, it is definitely true that the graph has automorphism group with the same cohomology as $\Sigma_{p}$. The lemma follows.

For an example of what we were trying to avoid in the proof of the above lemma, refer to the three examples given in the Figure 4 . The graphs pictured have an obvious $\mathbb{Z} / p$-symmetry given by rotation about the basepoint, which is indicated by a solid dot. But they have no dihedral flip, and their basepoint-preserving automorphism groups are all exactly $\mathbb{Z} / p$, where $p=5$ in the examples pictured and where obvious analogues exist for other odd primes. The ranks of the fundamental groups of the graphs pictured are $3 p-1,3 p$, and $2 p$, respectively. The last rank, $2 p$, is the lowest rank possible where one can have a graph with exactly $\mathbb{Z} / p$ symmetry.

Corollary 9. A vertex in the p-singular locus of $X_{n}$ has at most dihedral symmetry if its cohomology is the same as that of $D_{2 p}$ or $D_{2 p} \times \Sigma_{p}$, and vertices in the $p$-singular locus will never have exactly $\mathbb{Z} / p$ symmetry.

In the figures below, a dotted line or a hollow dot indicates that the given edge or vertex, respectively, does not have the indicated property. A solid dot, a solid line, or a 2-simplex with an $\mathrm{X}$ in it, means that the given vertex, edge, or 2-simplex, respectively, does have the indicated property.

By analyzing the $\mathbb{Z} / p$-invariant subforests of all of the graphs explicitly listed in the proof of Lemma 7, we can see what types of stabilizers higher dimensional simplices (rather than just vertices) have.

We will show that the simplices with at most dihedral symmetry will fall into two (exhaustive but not disjoint) categories. The first category consists of those that are listed in Figure 5. The second category consists of simplices whose maximal vertex (recall that $X_{n}$ is the realization of a poset) has the form $\Xi_{p} \vee \Gamma_{p-1}$ where $\Gamma_{p-1}$ is some basepointed graph with fundamental group of rank $p-1$, the wedge does not necessarily 
take place at the basepoint, and where the forest collapses of the simplex respect the $\mathbb{Z} / p$ action on $\Xi_{p}$.

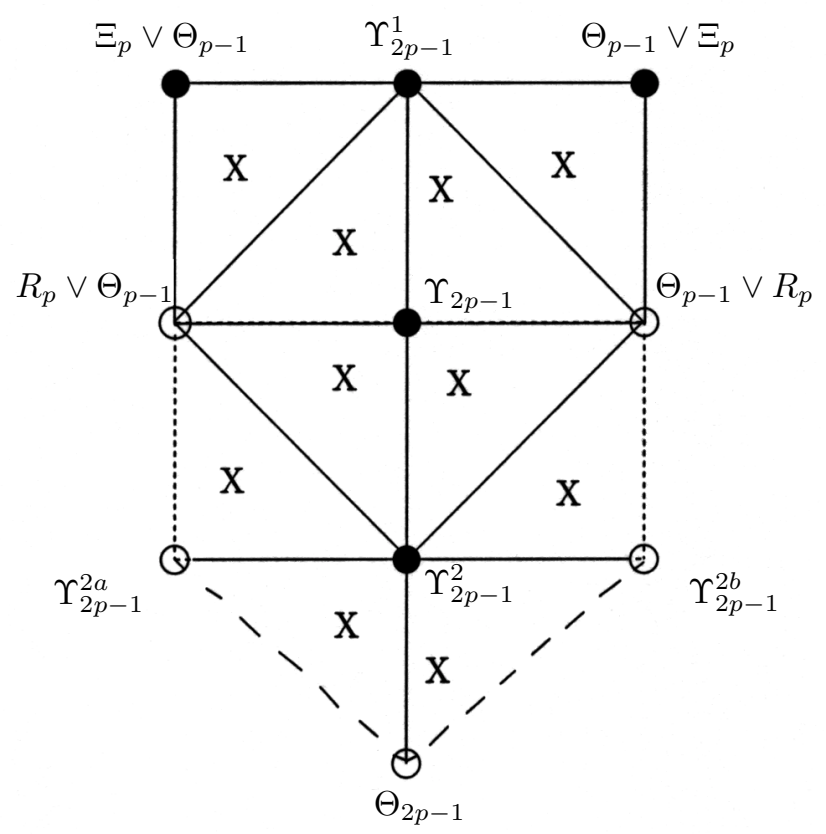

Figure 5. Some simplices with at most dihedral symmetry

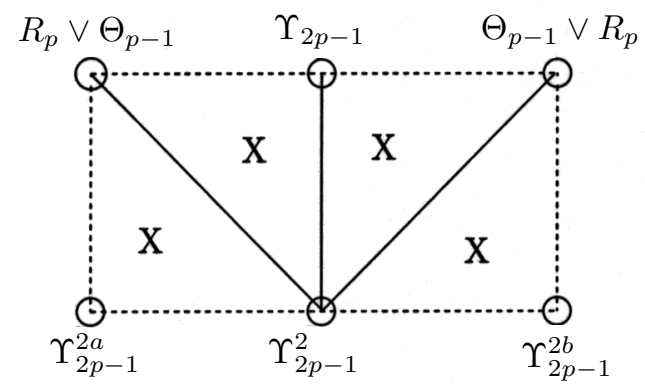

FIGURE 6. Simplices with exactly $\mathbb{Z} / p$ symmetry 
We will also show that the simplices listed in Figure 6 are the only ones with exactly $\mathbb{Z} / p$ symmetry.

Corollary 10. Let $p \geq 5$ be prime, $n=2 p-1$, and consider the $p$-singular locus of the spine $X_{n}$ of auter space.

- The only simplices with at most dihedral symmetry are either: (i) listed in Figure 5; or (ii) have maximal vertex of the form $\Xi_{p} \vee$ $\Gamma_{p-1}$.

- The only simplices with exactly $\mathbb{Z} / p$ symmetry are those listed in Figure 6.

Proof: We examine each of the graphs listed in Lemma 7 separately. By enumerating the $\mathbb{Z} / p$ invariant subforests of each of these graphs, one can list all of the simplices in the $p$-singular locus of $X_{n}$. We can ignore the graphs in Lemma 7 that do not have dihedral symmetry, as all of their symmetry comes from symmetric groups. When you collapse invariant subforests of these graphs, you still get graphs with symmetry coming from the symmetric group.

So we are left with analyzing the graphs from Lemma 7 with dihedral symmetry, which were:

- $\Theta_{p-1} \vee \Xi_{p}$. (There are actually two possibilities here as the enumeration in Lemma 7 did not specify basepoints. The central vertex of $\Xi_{p}$ could be attached to either the basepoint of $\Theta_{p-1}$ or the other vertex of $\Theta_{p-1}$.)

- $\Gamma_{p-1} \vee \Xi_{p}$, where $\Gamma_{p-1}$ is a basepointed graph with fundamental group of rank $p-1$ which has no $p$-symmetry (or where $\Gamma_{p-1}$ is $\Theta_{p-1}$ but the central vertex of $\Xi_{p}$ is attached to the midpoint of an edge of $\left.\Gamma_{p-1}\right)$.

- $\Upsilon_{2 p-1}$.

- $\Upsilon_{2 p-1}^{1}$.

- $\Upsilon_{2 p-1}^{2}$.

For the first two types of graphs, you can obtain simplices with dihedral symmetry by collapsing all of the spokes of $\Xi_{p}$ and/or any forest in the other graph of the wedge sum (either $\Theta_{p-1}$ or $\Gamma_{p-1}$ ). The resulting simplex with maximal vertex $\Theta_{p-1} \vee \Xi_{p}$ or $\Gamma_{p-1} \vee \Xi_{p}$ will clearly have at most dihedral symmetry, and will also just as clearly not give you a graph with exactly $\mathbb{Z} / p$ symmetry.

In a similar manner, simplices in the $p$-singular locus of $X_{n}$ with maximal vertex $\Upsilon_{2 p-1}$ or $\Upsilon_{2 p-1}^{1}$ are (exhaustively) listed in Figure 5 . Note that $\Upsilon_{2 p-1}$ can only be blown up (while still preserving the $\mathbb{Z} / p$ 
action so that we stay in the $p$-singular locus of $X_{n}$ ) in two ways, to either $\Upsilon_{2 p-1}^{1}$ or $\Upsilon_{2 p-1}^{2}$. The latter two graphs cannot be blown up at all.

Finally, the simplices with maximal vertex $\Upsilon_{2 p-1}^{2}$ are listed in Figure 5 or Figure 6 . Note that we can obtain edges and 2 -simplices with exactly $\mathbb{Z} / p$ symmetry, even though no actual vertex of $X_{n}$ has exactly $\mathbb{Z} / p$ symmetry. This is because you can choose subforests of $\Upsilon_{2 p-1}^{2}$ that do respect the dihedral "flip" of $\Upsilon_{2 p-1}^{2}$. In other words, this flip will not take the subforest to itself again. Hence the resulting simplex will just have symmetry group $\mathbb{Z} / p$. Last of all, note that you can also choose subforests of $\Upsilon_{2 p-1}^{2}$ which do respect the dihedral flip, and these give simplices with dihedral symmetry.

\section{The integral cohomology of the quotient never stabilizes}

We will prove Theorem 2 in this section. As in Section 4, all primes $p$ considered are assumed to be greater than or equal to 5 .

Lemma 11. For the rows $0 \leq s<2(p-1)$, the $E_{2}$ page of the spectral sequence (5) applied to calculate $H^{*}\left(\operatorname{Aut}\left(F_{2 p-1}\right) ; \mathbb{Z}_{(p)}\right)$ is given by

$$
E_{2}^{r, s}= \begin{cases}H^{r}\left(Q_{2 p-1} ; \mathbb{Z}_{(p)}\right) & s=0 \\ \mathbb{Z} / p & r=2 \text { and } s=4 k-2>0, k \in \mathbb{Z}^{+} \\ 0 & \text { otherwise. }\end{cases}
$$

Proof: As $E_{1}^{r, 0}$ is the cochain complex $C^{r}\left(Q_{2 p-1} ; \mathbb{Z}_{(p)}\right)$, it follows that $E_{2}^{r, 0}=H^{r}\left(Q_{2 p-1} ; \mathbb{Z}_{(p)}\right)$ as claimed above.

None of the simplices in $X_{2 p-1}$ contribute anything to the odd rows between 0 and $2(p-1)$ of the above spectral sequence, from Corollary 10 . Also from Corollary 10, the ones that contribute to rows of the form $4 k-2$, $k \in \mathbb{Z}^{+}$, are all listed in Figure 6 . Let $A$ be the subcomplex of $Q_{n}$ generated by all of the simplices pictured in Figure 6 and let $B$ be the subcomplex generated by just the simplices corresponding to dotted lines or hollow dots in Figure 6 . Then the row $s=4 k-2$ on the $E_{1}$ page of the spectral sequence is $C^{r}(A, B ; \mathbb{Z} / p)$. Examining Figure 6 we see that

$$
H^{r}(A, B ; \mathbb{Z} / p)= \begin{cases}\mathbb{Z} / p & r=2 \\ 0 & \text { otherwise. }\end{cases}
$$

Consequently the $E_{2}$ page is as claimed for the rows $s=4 k-2$. 
Our final task is to calculate the $E_{2}$ page for the rows $s=4 k$. Simplices in the $p$-singular locus of the spine with "at most dihedral symmetry" contribute to these rows. From Corollary 10, we have a characterization of such simplices. Define the subcomplex $M$ of the $p$-singular locus of the spine $X_{2 p-1}$ of auter space to be the subcomplex generated by simplices with "at most dihedral symmetry". More precisely, from Corollary 10, we know it is generated by the simplices corresponding to those in Figure 5 (i.e., corresponding in the sense that we are taking $M$ to be a subcomplex of the spine rather than its quotient and Figure 5 is a picture in the quotient) in addition to simplices whose maximal vertex has underlying graph of the form $\Xi_{p} \vee \Gamma_{p-1}$ (where the forest collapses in the simplices respect the $\mathbb{Z} / p$ action on $\Xi_{p}$ ). Recall that an $r$-simplex with at most dihedral symmetry contributes exactly one $\mathbb{Z} / p$ to $E_{1}^{r, 4 k}$, while all other simplices (those without dihedral or exactly $\mathbb{Z} / p$ symmetry) contribute nothing to this row.

Let $N$ be the subcomplex of $M$ generated by simplices in $M$ which do not have at most dihedral symmetry. Observe that none of the simplices in $N$ have at most dihedral symmetry. Also note that the row $E_{1}^{*, 4 k}$ is the relative cochain complex

$$
C^{*}\left(M / \operatorname{Aut}\left(F_{2 p-1}\right), N / \operatorname{Aut}\left(F_{2 p-1}\right) ; \mathbb{Z} / p\right) .
$$

Let $M^{\prime}$ be the subcomplex of $M$ generated by $N$ and by simplices whose maximal vertex is $\Upsilon_{2 p-1}^{2}$. Hence $M^{\prime}$ is the subcomplex consisting of $N$ and the bottom two thirds of Figure 5. There is an $\operatorname{Aut}\left(F_{2 p-1}\right)$-equivariant deformation retraction of $M$ onto $M^{\prime}$, given on the vertices of the poset by:

- Contracting the spokes of the graph $\Xi_{p}$ in $\Theta_{p-1} \vee \Xi_{p}$.

- Contracting the spokes of the graph $\Xi_{p}$ in $\Gamma_{p-1} \vee \Xi_{p}$, where $\Gamma_{p-1}$ has no $p$-symmetry.

- Contracting the $p$ outward radiating edges attached to the $p$-gon in the center of the graph $\Upsilon_{2 p-1}^{1}$. In the terminology used at the beginning of Section 4 while defining $\Upsilon_{2 p-1}^{1}$, we are contracting the edges $e_{i}$.

That it is a deformation retraction follows from the Poset Lemma in [10] attributed to Quillen.

As the homotopy retracting $M$ to $M^{\prime}$ is $\operatorname{Aut}\left(F_{2 p-1}\right)$-invariant, it descends to a deformation retraction of $M / \operatorname{Aut}\left(F_{2 p-1}\right)$ to $M^{\prime} / \operatorname{Aut}\left(F_{2 p-1}\right)$. Hence the relative cohomology groups

$$
H^{*}\left(M / \operatorname{Aut}\left(F_{2 p-1}\right), N / \operatorname{Aut}\left(F_{2 p-1}\right) ; \mathbb{Z} / p\right)
$$


and

$$
H^{*}\left(M^{\prime} / \operatorname{Aut}\left(F_{2 p-1}\right), N / \operatorname{Aut}\left(F_{2 p-1}\right) ; \mathbb{Z} / p\right)
$$

are isomorphic. Now referring to Figure 5 , we see that

$$
H^{t}\left(M^{\prime} / \operatorname{Aut}\left(F_{2 p-1}\right), N / \operatorname{Aut}\left(F_{2 p-1}\right) ; \mathbb{Z} / p\right)=0
$$

for all $t$ because we can contract all of the simplices in $M^{\prime} / \operatorname{Aut}\left(F_{2 p-1}\right)$ uniformly into $N / \operatorname{Aut}\left(F_{2 p-1}\right)$.

An immediate consequence is

Proof of Theorem 2: From [6], if $m \geq 8 k+3$, then the standard map

$$
H^{4 k}\left(\operatorname{Aut}\left(F_{m+1}\right) ; \mathbb{Z}\right) \rightarrow H^{4 k}\left(\operatorname{Aut}\left(F_{m}\right) ; \mathbb{Z}\right)
$$

is an isomorphism. Observe that $H^{4 k}\left(\operatorname{Aut}\left(F_{8 k+3}\right) ; \mathbb{Z}\right)=H^{4 k}\left(\operatorname{Aut}\left(F_{\infty}\right) ; \mathbb{Z}\right)$ is a finitely generated abelian group. If it contains a torsion free summand isomorphic to $\mathbb{Z}$, then we are done and $H^{4 k}\left(\operatorname{Aut}\left(F_{\infty}\right) ; \mathbb{Q}\right) \neq 0$. Otherwise, choose a prime $q$ such that $2 q-1 \geq 8 k+3$ and so that for all primes $p \geq q$ there is no $p$-torsion in $H^{4 k}\left(\operatorname{Aut}\left(F_{8 k+3}\right) ; \mathbb{Z}\right)$. We will show that $H^{4 k+1}\left(Q_{2 p-1} ; \mathbb{Z}\right)$ has $p$-torsion for all primes $p \geq q$, which will prove the theorem.

Let $p \geq q$. From the lemma above, if we use the standard equivariant spectral sequence to calculate $H^{*}\left(\operatorname{Aut}\left(F_{2 p-1}\right) ; \mathbb{Z}_{(p)}\right)$, then a class $\alpha \in$ $E_{1}^{2,4 k-2}$ in the $E_{1}$-page survives at least until the $E_{4 k-1}$-page.

Because $H^{4 k}\left(\operatorname{Aut}\left(F_{2 p-1}\right) ; \mathbb{Z}\right)$ has no $p$-torsion and $H^{4 k}\left(\operatorname{Aut}\left(F_{2 p-1}\right)\right.$; $\mathbb{Q})=0$, we have $H^{4 k}\left(\operatorname{Aut}\left(F_{2 p-1}\right) ; \mathbb{Z}_{(p)}\right)=0$. Hence the class $\alpha \in E_{1}^{2,4 k-2}$ cannot survive to the $E_{\infty}$ page. It follows that there is $p$-torsion in $E_{4 k-1}^{4 k+1,0}$. Recall that $E_{1}^{r, 0}$ corresponds to the cellular chain complex with $\mathbb{Z}_{(p)}$ coefficients for $Q_{2 p-1}$. The $p$-torsion in $E_{4 k-1}^{4 k+1,0}$, therefore, would have to have been created when going from the $E_{1}$ to $E_{2}$ pages, because any of the torsion above the horizontal axis of the spectral sequence could not map onto a torsion free element on the horizontal axis. So $H^{4 k+1}\left(Q_{2 p-1} ; \mathbb{Z}_{(p)}\right)$ has $p$-torsion, and thus $H^{4 k+1}\left(Q_{2 p-1} ; \mathbb{Z}\right)$ has $p$-torsion.

\section{References}

[1] G. Baumslag and T. Taylor, The centre of groups with one defining relator, Math. Ann. 175 (1968), 315-319.

[2] K. S. Brown, "Cohomology of groups", Graduate Texts in Mathematics 87, Springer-Verlag, New York-Berlin, 1982.

[3] M. Culler and K. Vogtmann, Moduli of graphs and automorphisms of free groups, Invent. Math. 84(1) (1986), 91-119. 
[4] H. H. Glover And G. Mislin, On the $p$-primary cohomology of $\operatorname{Out}\left(F_{n}\right)$ in the $p$-rank one case, J. Pure Appl. Algebra 153(1) (2000), 45-63.

[5] A. HATCHER, Homological stability for automorphism groups of free groups, Comment. Math. Helv. 70(1) (1995), 39-62.

[6] A. Hatcher and K. Vogtmann, Cerf theory for graphs, J. London Math. Soc. (2) 58(3) (1998), 633-655.

[7] A. Hatcher and K. Vogtmann, Rational homology of $\operatorname{Aut}\left(F_{n}\right)$, Math. Res. Lett. 5(6) (1998), 759-780.

[8] C. A. Jensen, Cohomology of $\operatorname{Aut}\left(F_{n}\right)$, Cornell University Ph. D. dissertation, Ithaca, New York (1998).

[9] C. A. Jensen, Cohomology of $\operatorname{Aut}\left(F_{n}\right)$ in the $p$-rank two case, J. Pure Appl. Algebra 158(1) (2001), 41-81.

[10] S. Krstić And K. Vogtmann, Equivariant outer space and automorphisms of free-by-finite groups, Comment. Math. Helv. 68(2) (1993), 216-262.

[11] H. Minkowski, Zur Theorie der positiven quadratischen Formen, Crelles J. 101 (1887), 196-202.

[12] J. Smillie And K. Vogtmann, A generating function for the Euler characteristic of Out $\left(F_{n}\right)$, in: "Proceedings of the Northwestern conference on cohomology of groups" (Evanston, Ill., 1985), J. Pure Appl. Algebra 44(1-3) (1987), 329-348.

[13] J. Smillie and K. Vogtmann, Automorphisms of graphs, $p$-subgroups of $\operatorname{Out}\left(F_{n}\right)$ and the Euler characteristic of $\operatorname{Out}\left(F_{n}\right), J$. Pure Appl. Algebra 49(1-2) (1987), 187-200.

[14] R. G. Swan, The $p$-period of a finite group, Illinois J. Math. 4 (1960), 341-346.

Department of Mathematics

University of New Orleans

New Orleans, LA 70148

U.S.A.

E-mail address: jensen@math.uno.edu 